\title{
Abundant Interaction Solutions of Sine-Gordon Equation
}

\author{
DaZhao Lï, ${ }^{1}$ YanYing Cui, ${ }^{2}$ ChangHe Liu, ${ }^{1}$ and ShangWen Wu ${ }^{1}$ \\ ${ }^{1}$ School of Science, Beijing University of Civil Engineering and Architecture, Beijing 100044, China \\ ${ }^{2}$ Department of Basic Science, Gengdan Institute of Beijing University of Technology, \\ Beijing 101301, China \\ Correspondence should be addressed to DaZhao Lü, lvdazhao86@163.com
}

Received 15 July 2012; Accepted 2 October 2012

Academic Editor: Junjie Wei

Copyright (C) 2012 DaZhao Lü et al. This is an open access article distributed under the Creative Commons Attribution License, which permits unrestricted use, distribution, and reproduction in any medium, provided the original work is properly cited.

With the help of computer symbolic computation software (e.g., Maple), abundant interaction solutions of sine-Gordon equation are obtained by means of a constructed Wronskian form expansion method. The method is based upon the forms and structures of Wronskian solutions of sine-Gordon equation, and the functions used in the Wronskian determinants do not satisfy linear partial differential equations. Such interaction solutions are difficultly obtained via other methods. And the method can be automatically carried out in computer.

\section{Introduction}

To search for the exact solutions of the soliton equations, many different methods were developed, such as Bäcklund transformation [1], Darboux transformation [2], the inverse scattering transformation [3], bilinear method [4], the special mapping relations method [5], and Wronskian technique [6]. Among them, the Wronskian is a powerful tool. One advantage of the Wronskian formulation is that solutions may be verified by direct substitution into the bilinear equations or by inductive use of its bilinear Bäcklund transformations. The key to constructing Wronskian solutions is the suitable choices and determinations of the functions used in the Wronskian determinants $\phi_{i}(1 \leqslant i \leqslant N)$. Generally, these functions $\phi_{i}(1 \leqslant i \leqslant N)$ satisfy the linear partial differential equations.

In mathematics, a natural question to ask is whether there exist solutions in the Wronskian form if the functions used in the Wronskian determinants $\phi_{i}(1 \leqslant i \leqslant N)$ do not satisfy linear partial differential equations. How to obtain Wronskian form solutions if they exist? 
In physics, the solitary waves, which can pass through each other and preserve their individual shapes and velocities with only a small change in their phases after the collision with other solitary waves, were called solitons to indicate these remarkable quasiparticle properties. Since the solitary wave is a limited case of the elliptic function (special function) wave, what happens after a elliptic wave collides with other elliptic waves?

So it is very interesting in both mathematics and physics to search for the interaction solutions between elliptic wave and solitary wave, which will be more helpful to a better understanding of objective laws described by the nonlinear evolution equation. However, up to now, there are few works in the previous literatures to study the interaction behaviors between elliptic wave and solitary wave, or between elliptic wave and other elliptic wave. It is very difficult to search for such interactions although solitary wave is a limited case of elliptic wave. The reason is that the ansatz of the interaction solutions between elliptic wave and solitary wave is difficult to be determined.

In this paper, we present a Wronskian form expansion method to construct new interaction solutions between elliptic wave and solitary wave to the sine-Gordon equation. Our method is based upon forms and structures of Wronskian solutions of the sine-Gordon equation, but it does not require that the functions used in the Wronskian determinants satisfy linear partial differential equations.

\section{Wronskian Form Expansion Method and Abundant Interaction Solutions}

The sine-Gordon equation (SG) reads

$$
u_{x t}=\sin u
$$

It has a wide range of applications in almost all the branches of physics and other scientific fields [1-9], not only in relativistic quantum field theories [10] but also in solid-state physics [11], nonlinear optics [12], the theory of long Josephson junctions [13] and liquid crystals [14], and so forth.

The SG has bilinear form

$$
D_{x} D_{t} f \cdot f=\frac{1}{2}\left(f^{2}-f^{* 2}\right)
$$

under a variable transformation

$$
u=2 i \ln \frac{f^{*}}{f}
$$

where $f^{*}$ is the complex conjugate of $f$ and $D$ is the well-known Hirota's bilinear operators, defined by

$$
\left.D_{x}^{m} D_{t}^{n}(a \cdot b) \equiv\left(\frac{\partial}{\partial x}-\frac{\partial}{\partial x^{\prime}}\right)^{m}\left(\frac{\partial}{\partial t}-\frac{\partial}{\partial t^{\prime}}\right)^{n} a(x, t) b\left(x^{\prime}, t^{\prime}\right)\right|_{\substack{x=x^{\prime} \\ t=t^{\prime}}}
$$


To take advantage of the Wronskian technique, one can use the compact Freeman and Nimmo's notation [6]

$$
(\widehat{N-1})=W\left(\phi_{1}, \phi_{2}, \ldots, \phi_{N}\right)=\left|\begin{array}{cccc}
\phi_{1} & \phi_{1}^{(1)} & \cdots & \phi_{1}^{(N-1)} \\
\cdots & \cdots & \cdots & \cdots \\
\phi_{N} & \phi_{N}^{(1)} & \cdots & \phi_{N}^{(N-1)}
\end{array}\right|
$$

where

$$
\phi_{i}^{(j)}=\frac{\partial^{j}}{\partial x^{j}} \phi_{i}, \quad j \geqslant 1,(1 \leqslant i \leqslant N) .
$$

If $f=(\widehat{N-1})$ and the functions used in the Wronskian determinants $\phi_{i}(1 \leqslant i \leqslant N)$ satisfy the linear partial differential equations [6]

$$
\phi_{i, x x}=k_{i}^{2} \phi_{i}, \quad \phi_{i, t t}=\left(16 k_{i}^{2}\right)^{-1} \phi_{i}
$$

or

$$
\phi_{i, x}=k_{i} \phi_{i}^{*}, \quad \phi_{i, t}=\left(4 k_{i}\right)^{-1} \phi_{i}^{*},
$$

where $k_{i}$ are arbitrary real constants, then such an $f$ solves the bilinear SG equation (2.2). Therefore, $u=2 i \ln f^{*} / f$ in (2.3) gives an exact solution of the SG equation (2.1).

A general solution to the system of linear partial differential equation (2.7) is given by

$$
\phi_{i}=\alpha_{i} \exp \left(k_{i} x+\frac{1}{4 k_{i}} t\right)+i \beta_{i} \exp \left(-k_{i} x-\frac{1}{4 k_{i}} t\right)
$$

where $\alpha_{i}, \beta_{i}$, and $k_{i}$ are arbitrary real constants.

In particular, for $N=2, \alpha_{i}=\beta_{i}$, and $f=f_{r}+i f_{I}$, the following

$$
\begin{aligned}
u & =2 i \ln \frac{f^{*}}{f}=4 \arctan \frac{f_{I}}{f_{r}} \\
& =4 \arctan \frac{\left(k_{1}+k_{2}\right) \sinh \left(\left(k_{1}-k_{2}\right)\left(x-\left(4 k_{1} k_{2}\right)^{-1} t\right)\right)}{\left(k_{1}-k_{2}\right) \cosh \left(\left(k_{1}+k_{2}\right)\left(x+\left(4 k_{1} k_{2}\right)^{-1} t\right)\right)}
\end{aligned}
$$

is a solution of the SG (2.1).

However, if we take the functions $\phi_{1}$ and $\phi_{2}$ in the form

$$
\phi_{1}=\operatorname{dn} \xi_{1}, \quad \phi_{2}=\sinh \left(\xi_{2}+\theta i\right), \quad \xi_{i}=k_{i} x+l_{i} t, \quad i=1,2,
$$


where $\operatorname{dn} \xi_{1} \equiv \operatorname{dn}\left(\xi_{1}, m\right)$ is Jacobi elliptic function with the modulus $m$, and $\theta$ is arbitrary real constant, then, the following

$$
\begin{gathered}
u^{\prime}=2 i \ln \frac{f^{*}}{f}=4 \arctan \frac{f_{I}}{f_{r}} \\
=4 \arctan \frac{\sin \theta\left(m^{2} k_{1} \operatorname{sn} \xi_{1} \operatorname{cn} \xi_{1} \cosh \xi_{2}+k_{2} \operatorname{dn} \xi_{1} \sinh \xi_{2}\right)}{\cos \theta\left(m^{2} k_{1} \operatorname{sn} \xi_{1} \operatorname{cn} \xi_{1} \sinh \xi_{2}+k_{2} \operatorname{dn} \xi_{1} \cosh \xi_{2}\right)} \\
\xi_{i}=k_{i} x+l_{i} t, \quad i=1,2
\end{gathered}
$$

is not a solution to the SG equation (2.1). The reason is that Jacobi elliptic function $\mathrm{dn} \xi_{1}$ in (2.10) does not satisfy the linear partial differential equations (2.7) or other various linear generalizations.

Although the above $u^{\prime}$ in (2.11) is not a solution to the SG equation (2.1), we may take advantage of its form and structure.

In the following steps, we will show our new Wronskian form expansion method.

Step 1 (suppose Wronskian form solution). First, we take the functions $\phi_{1}$ and $\phi_{2}$ in the form

$$
\phi_{1}=\operatorname{dn} \xi_{1}, \quad \phi_{2}=\sinh \left(\xi_{2}+\theta i\right), \quad \xi_{i}=k_{i} x+l_{i} t, \quad i=1,2
$$

Then we may suppose that the SG equation (2.1) has the following Wronskian form solution, which corresponds to $u^{\prime}$ in (2.11):

$$
\begin{gathered}
u=4 \arctan \frac{a_{1} \operatorname{sn} \xi_{1} \operatorname{cn} \xi_{1} \cosh \xi_{2}+a_{2} \operatorname{dn} \xi_{1} \sinh \xi_{2}}{b_{1} \operatorname{sn} \xi_{1} \operatorname{cn} \xi_{1} \sinh \xi_{2}+b_{2} \operatorname{dn} \xi_{1} \cosh \xi_{2}} \\
\xi_{i}=k_{i} x+l_{i} t, \quad i=1,2,
\end{gathered}
$$

where $a_{i}, b_{i}, k_{i}$ and $l_{i}, i=1,2$. are the unknowns to be determined later.

The purpose of such an supposition is to adjust coefficients of the form solution so that $u$ in (2.13) solves the SG equation (2.1). The ansatz $u$ in (2.13) is the suitable choices and determinations to obtain the interaction solutions between elliptic wave and solitary wave. If the ansatz is too general, then computations are very complicated, hard, and not practical. If the ansatz is too special, then the interaction solutions are possible to be left out and not discovered.

Step 2 (lead to the set of algebraic equations). Now substitute (2.13) into (2.1), and set all coefficients of the like powers of the resulting system's numerator to be zero; then get a set of algebraic equations with respect to the unknowns $k_{i}, l_{i}, a_{i}, b_{i}(i=1,2)$. We can determine these unknowns through solving the set of algebraic equations by using elimination methods [15]. 


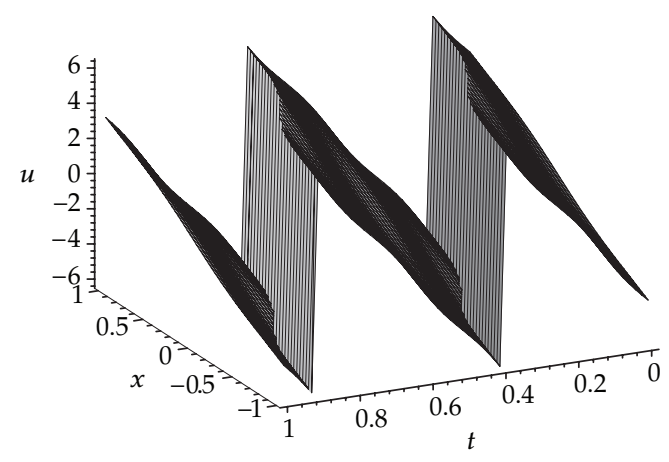

(a) $m=0.2$.

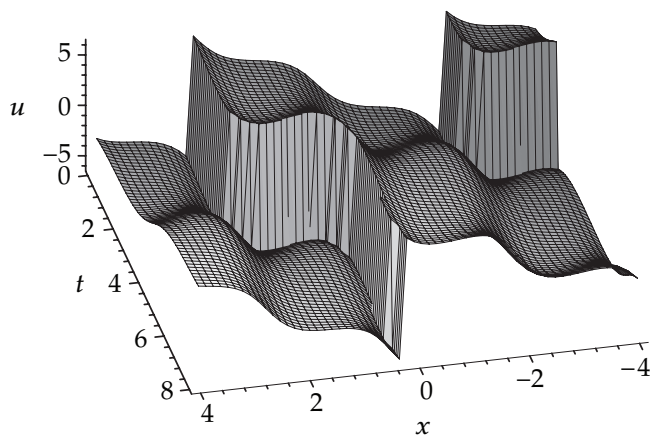

(b) $m=0.8$.

Figure 1: Two special cases of interaction solution $u_{1}$.

Step 3 (obtain abundant interaction solutions). At last, substituting the value of $k_{i}, l_{i}, a_{i}$, $b_{i}$ into (2.13), we can obtain the following new type of interaction solution between Jacobi elliptic function and trigonometric function to the SG equation (2.1):

$$
\begin{gathered}
u_{1}=4 \arctan \frac{m \operatorname{sn} \xi_{1} \mathrm{cn} \xi_{1} \cos \xi_{2}+\mathrm{dn} \xi_{1} \sin \xi_{2}}{m \operatorname{sn} \xi_{1} \mathrm{cn} \xi_{1} \sin \xi_{2}+\mathrm{dn} \xi_{1} \cos \xi_{2}}, \\
\xi_{1}=k_{1} x-\left(4 m^{2} k_{1}\right)^{-1} t \\
\xi_{2}=\sqrt{1-m^{2}}\left(k_{1} x+\left(4 m^{2} k_{1}\right)^{-1} t\right)
\end{gathered}
$$

and travelling wave solution to the SG equation (2.1):

$$
\begin{gathered}
u_{2}=4 \arctan \frac{m \operatorname{sn} \xi \operatorname{cn} \xi}{\operatorname{dn} \xi} \\
\xi=k x-(4 k)^{-1} t .
\end{gathered}
$$

Note: All of the solutions presented in this paper have been verified with respect to the original SG equation (2.1) by virtue of Maple. See the Appendix.

Figure 1 plots two cases of interaction solution $u_{1}$ with the same parameter select $k_{1}=$ 1, grid $=[60,60]$.

Similarly, let us fix

$$
\phi_{1}=1+\operatorname{dn} \xi_{1}, \quad \phi_{2}=\sinh \left(\xi_{2}+\theta i\right)
$$


in our Wronskian form expansion method, and by making use of the previous steps, we get

$$
\begin{gathered}
u_{3}=4 \arctan \frac{\sinh \xi_{2} \mathrm{dn} \xi_{1}}{\sqrt[4]{1-m^{2}} \cosh \xi_{2}}, \\
\xi_{1}=l_{1}^{-1}\left(1+\sqrt{1-m^{2}}\right)^{-2} x+l_{1} t \\
\xi_{2}=\sqrt[4]{1-m^{2}}\left(-l_{1}^{-1}\left(1+\sqrt{1-m^{2}}\right)^{-2} x+l_{1} t\right), \\
u_{4}=4 \arctan \frac{\sin \xi_{2} \mathrm{dn} \xi_{1}}{\sqrt[4]{1-m^{2}} \cos \xi_{2}}, \\
\xi_{1}=l_{1}^{-1}\left(\sqrt{1-m^{2}}-1\right)^{-2} x+l_{1} t \\
\xi_{2}=\sqrt[4]{1-m^{2}}\left(-l_{1}^{-1}\left(\sqrt{1-m^{2}}-1\right)^{-2} x+l_{1} t\right), \\
u_{5}=4 \arctan \frac{\sqrt[4]{1-m^{2}} \sinh \xi_{2}}{\cosh _{2} \mathrm{dn} \xi_{1}}, \\
\xi_{1}=l_{1}^{-1}\left(1+\sqrt{1-m^{2}}\right)^{-2} x+l_{1} t \\
\xi_{2}=\sqrt[4]{1-m^{2}}\left(-l_{1}^{-1}\left(1+\sqrt{1-m^{2}}\right)^{-2} x+l_{1} t\right), \\
\xi_{2} \sqrt{-2+2 \sqrt{1-m^{2}}+m^{2}}\left(l_{1}^{-1}\left(1+\sqrt{1-m^{2}}\right)^{-2} x+l_{1} t\right) . \\
u_{6}=4 \arctan \frac{\left(\mathrm{dn} \xi_{1}+\sqrt[4]{1-m^{2}}\right) \sinh \xi_{2}}{\left(\mathrm{dn} \xi_{1}-\sqrt[4]{1-m^{2}}\right) \cosh \xi_{2}},
\end{gathered}
$$

Taking

$$
\phi_{1}=1+\operatorname{sn} \xi_{1}, \quad \phi_{2}=\sinh \left(\xi_{2}+\theta i\right)
$$

we gain

$$
\begin{aligned}
& u_{7}=4 \arctan \frac{\left(\sqrt{m} \mathrm{sn} \xi_{1}+1\right) \sin \xi_{2}}{\left(\sqrt{m} \mathrm{sn} \xi_{1}-1\right) \cos \xi_{2}}, \\
& \xi_{1}=\frac{1}{4} l_{1}^{-1} m^{-1} x+l_{1} t, \\
& \xi_{2}=\frac{1}{8}(1-m)\left(l_{1}^{-1} m^{-1} x-4 l_{1} t\right),
\end{aligned}
$$


Journal of Applied Mathematics

$$
\begin{aligned}
& u_{8}=4 \arctan \frac{\left(\sqrt{m} \mathrm{sn} \xi_{1}+1\right) \sinh \xi_{2}}{\left(\sqrt{m} \mathrm{sn} \xi_{1}-1\right) \cosh \xi_{2}}, \\
& \xi_{1}=l_{1}^{-1}(1+m)^{-2} x+l_{1} t, \\
& \xi_{2}=\frac{1}{2}(1-m)\left(l_{1}^{-1}(1+m)^{-2} x-l_{1} t\right) .
\end{aligned}
$$

In general, we take the functions $\phi_{1}$ and $\phi_{2}$ in the form

$$
\phi_{1}=1+\operatorname{sn}\left(\xi_{1}, m_{1}\right), \quad \phi_{2}=\operatorname{sn}\left(\xi_{2}+\theta i, m_{2}\right) .
$$

Then we obtain the corresponding interaction solution between two Jacobi elliptic functions with two different moduli $m_{1}$ and $m_{2}$ :

$$
\begin{gathered}
u_{9}=4 \arctan \frac{\sqrt{1-m_{2}^{2}} \operatorname{sn}\left(\xi_{2}, m_{2}\right)\left(\sqrt{m_{1}} \operatorname{sn}\left(\xi_{1}, m_{1}\right)-1\right)}{\operatorname{cn}\left(\xi_{2}, m_{2}\right) \operatorname{dn}\left(\xi_{2}, m_{2}\right)\left(\sqrt{m_{1}} \operatorname{sn}\left(\xi_{1}, m_{1}\right)+1\right)}, \\
\xi_{1}=k_{1} x+\frac{\left(m_{2}^{2}-1\right) t}{k_{1}\left(m_{1}^{2} m_{2}^{2}+2 m_{2}^{2} m_{1}+m_{2}^{2}-4 m_{1}\right)}, \\
\xi_{2}=\frac{1-m_{1}}{2 \sqrt{1-m_{2}^{2}}}\left(k_{1} x-\frac{\left(m_{2}^{2}-1\right) t}{k_{1}\left(m_{1}^{2} m_{2}^{2}+2 m_{2}^{2} m_{1}+m_{2}^{2}-4 m_{1}\right)}\right) .
\end{gathered}
$$

Choosing

$$
\phi_{1}=1+\operatorname{dn}\left(\xi_{1}, m_{1}\right), \quad \phi_{2}=\operatorname{dn}\left(\xi_{2}+\theta i, m_{2}\right) .
$$

We have

$$
\begin{gathered}
u_{10}=4 \arctan \frac{\operatorname{dn}\left(\xi_{2}, m_{2}\right)\left(\operatorname{dn}\left(\xi_{1}, m_{1}\right)+\sqrt[4]{1-m_{1}^{2}}\right)}{m_{2} \operatorname{sn}\left(\xi_{2}, m_{2}\right) \operatorname{cn}\left(\xi_{2}, m_{2}\right)\left(\operatorname{dn}\left(\xi_{1}, m_{1}\right)-\sqrt[4]{1-m_{1}^{2}}\right)}, \\
\xi_{1}=\frac{1}{2} m_{2}\left(-\frac{x \sqrt{2 \sqrt{1-m_{1}^{2}}-2+m_{1}^{2}}}{l_{2}\left(\sqrt{1-m_{1}^{2}}\left(4 m_{2}^{2}-2\right)+2-m_{1}^{2}\right)}+\frac{4 l_{2} t}{\sqrt{2 \sqrt{1-m_{1}^{2}}-2+m_{1}^{2}}}\right), \\
\xi_{2}=\frac{x\left(2 \sqrt{1-m_{1}^{2}}-2+m_{1}^{2}\right)}{4 l_{2}\left(\sqrt{1-m_{1}^{2}}\left(4 m_{2}^{2}-2\right)+2-m_{1}^{2}\right)}+l_{2} t .
\end{gathered}
$$




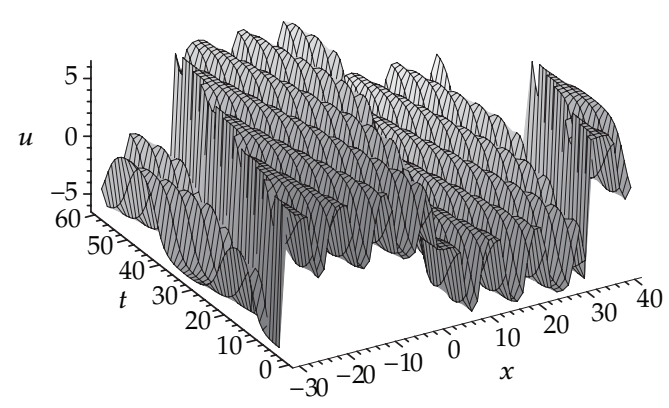

(a) $u_{9}: m_{1}=0.9, m_{2}=0.1, k_{1}=1$.

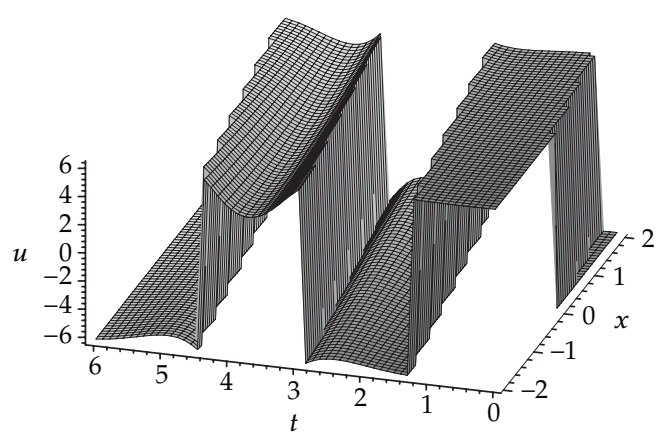

(b) $u_{10}: m_{1}=0.8, m_{2}=0.2, l_{2}=1$.

Figure 2: Two special cases of the solutions $u_{9}(\mathrm{a})$ and $u_{10}(\mathrm{~b})$.

Figure 2 shows two special structures of the solutions $u_{9}$ and $u_{10}$, respectively, where grid $=$ $[60,60]$.

In particular, we can easily obtain many travelling wave solutions via our Wronskian form expansion method. For instance, only choosing the function $\phi_{1}=1+\operatorname{sn}(\xi+\theta I)$ as the simplest example, we can get many travelling wave solutions; the following are some travelling wave solutions of them:

$$
\begin{gathered}
u_{11}=4 \arctan \frac{\mathrm{cn} \xi \mathrm{dn} \xi}{\sqrt{1-m^{2}} \mathrm{sn} \xi} \\
\xi=k x+\frac{1}{4 k m^{2}} t \\
u_{12}=-4 \arctan \frac{\mathrm{dn} \xi}{\sqrt{m} \mathrm{cn} \xi} \\
\xi=k x-\frac{1}{k(1+m)^{2}} t \\
u_{13}=4 \arctan \frac{\mathrm{cn} \xi \operatorname{dn} \xi}{1-(m+1) \operatorname{sn} \xi+m \mathrm{sn}^{2} \xi} \\
\xi=k x+\frac{1}{4 k m} t \\
u_{14}=4 \arctan \frac{\sqrt[4]{4 m} \mathrm{cn} \xi \mathrm{dn}^{2}}{\sqrt{1+m}\left(-1+m \mathrm{sn}^{2} \xi\right)} \\
\xi=k x-\frac{(1-\sqrt{m})^{2}}{k(1-m)^{2}(1+\sqrt{m})^{2}} t .
\end{gathered}
$$

Remark 2.1. It is easily seen that our method is more powerful than the methods searching for traveling wave solutions, such as the Jacobi elliptic function method [16, 17], the tanh method [18], the sine-cosine method [19], and Exp-function method [20], Fan subequation 
method [21], F-expansion method [22], Riccati method [23], Hyperbolic function method [24], and $G^{\prime} / G$ expansion method [25]. It is difficult to obtain the interaction solutions between two different kinds of functions by the methods [16-25] and the classical methods, such as the inverse scattering transformation, Bäcklund transformation, Darboux transformation, bilinear method, perturbation method, and the dressing method.

Remark 2.2. Recently, Ma and Lee presented a Frobenius decompositions method [26] and a transformed rational function method [27] for solving nonlinear differential equations. Although their methods are very good and our method cannot recover the solutions obtained by Ma's methods $[26,27]$, new types of the interaction solutions obtained by us cannot be found by Ma's methods.

Remark 2.3. In fact, we can choose other types of the functions to get other new types of interaction solutions in our Wronskian form expansion method. For simplification, we omit them in the paper. We can also generate Wronskian solutions of higher order by inputting more special functions in the Wronskian formulation. But, higher the order is, the more difficult the computation of nonlinear algebraic equations by using elimination methods [15] is.

Remark 2.4. Although our method seems complicated, the procedure can be automatically carried out in computer symbolic computation software (e.g., Maple), which makes the process simple.

\section{Conclusions and Discussion}

In this paper, only on the basis of the forms and structures of Wronskian solutions to the SG equation, we have presented the Wronskian form expansion method to construct a few new types of interaction solutions which we have never seen before within our knowledge, and the functions used in the Wronskian determinants don't satisfy the linear partial differential equations. Our exact solutions indicate that the solitary wave can preserve its shape and velocity with only a small change in its phase after the collision with elliptic wave, and the elliptic wave can also preserve its shape and speed upon collision with other elliptic wave. The method can be applied to other soliton equations.

Our results with Jacobi elliptic functions, which satisfy the nonlinear differential equations, suggest that it seems reasonable to believe that there exist the nonlinear Wronskian condition equations to the SG equation, further, one can obtain more general new types of multi-interaction solutions with Jacobi elliptic functions and other functions to the SG equation. How to find these nonlinear Wronskian condition equations will be an interesting topic. A general theory on Wronskian technique of the soliton equations still needs to be further explored.

\section{Appendix}

As claimed, all of our solutions in this paper have been verified by the use of Maple. The following is an example. For instance, the solution $u_{1}$, that is, (2.14), can be straightforwardly verified with the Maple program below, in which $\mathrm{sn} \xi, \mathrm{cn} \xi, \mathrm{dn} \xi$ are the user-defined verification functions. One may also use Maple's default command functions JacobiSN $(\xi, m)$, $\operatorname{JacobiCN}(\xi, m)$, JacobiDN $(\xi, m)$ instead of the user-defined functions $\operatorname{sn} \xi, \mathrm{cn} \xi$, dn $\xi$. The 
results of output indicate that $u_{1}$, that is, (2.14), is indeed a particular solution for the original SG equation (2.1):

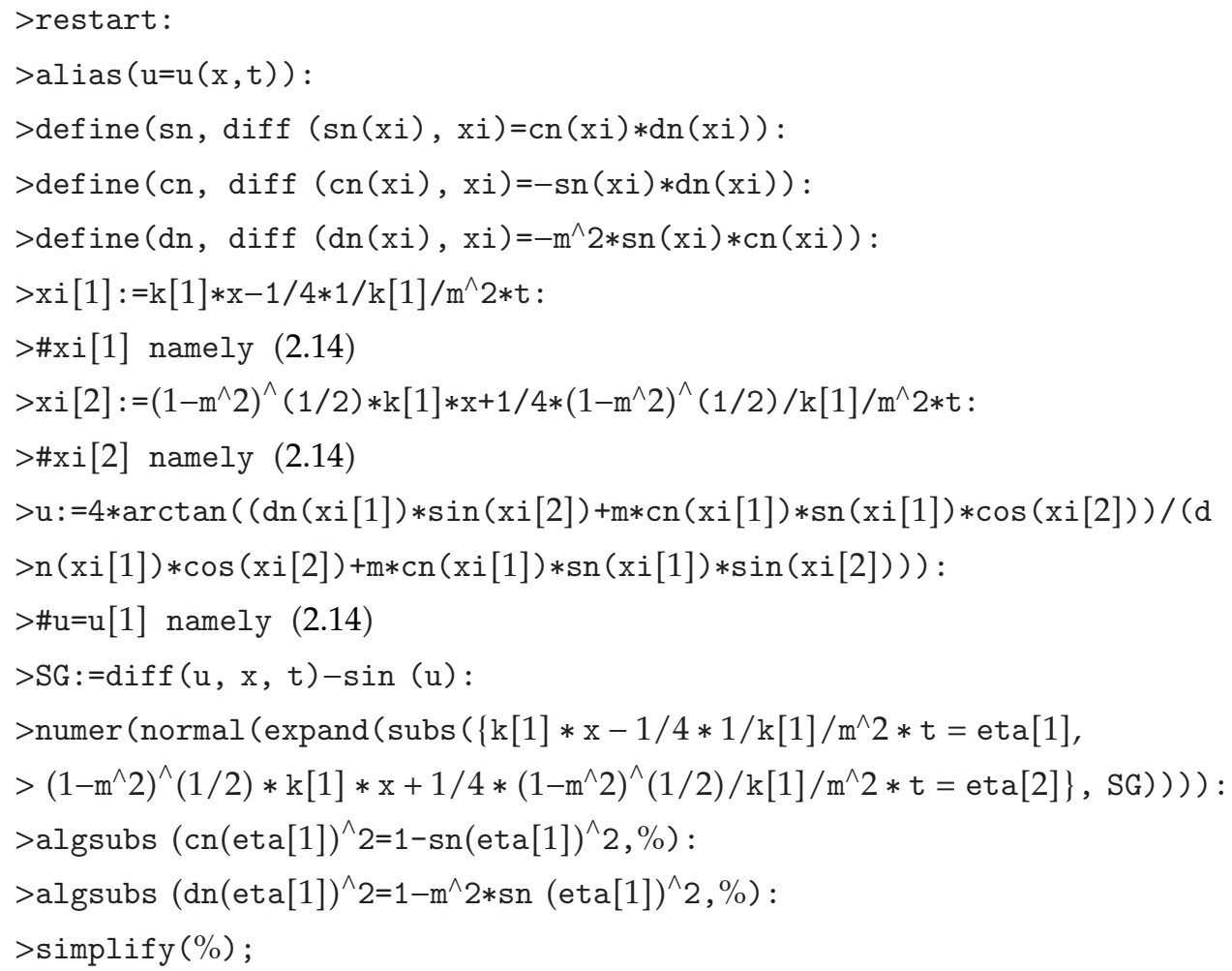

\section{Acknowledgment}

The first and third authors would like to acknowledge the financial support of the project for Academic Human Resources Development in Institutions of Higher Learning Under the Jurisdiction of Beijing Municipality (PHR201107123).

\section{References}

[1] A. Seeger, H. Donth, and A. Kochendörfer, "Theorie der Versetzungen in eindimensionalen Atomreihen. III. Versetzungen, Eigenbewegungen und ihre Wechselwirkung," Zeitschrift für Physik. C, vol. 134, pp. 173-193, 1953.

[2] V. B. Matveev and M. A. Salle, Darboux Transformations and Solitons, Springer Series in Nonlinear Dynamics, Springer, Berlin, Germany, 1991.

[3] M. J. Ablowitz, D. J. Kaup, A. C. Newell, and H. Segur, "Method for solving the sine-Gordon equation," Physical Review Letters, vol. 30, pp. 1262-1264, 1973.

[4] R. Hirota and J. Satsuma, "A simple structure of superposition formula of the backlund transformation," Journal of the Physical Society of Japan, vol. 45, no. 5, pp. 1741-1750, 1978.

[5] S. Y. Lou, H. C. Hu, and X. Y. Tang, "Interactions among periodic waves and solitary waves of the $(\mathrm{N}+1)$-dimensional sine-Gordon field," Physical Review E, vol. 71, no. 3, Article ID 036604, 8 pages, 2005.

[6] J. J. C. Nimmo and N. C. Freeman, "The use of Bäcklund transformations in obtaining N-soliton solutions in Wronskian form," Journal of Physics A, vol. 17, no. 7, pp. 1415-1424, 1984. 
[7] A. V. Bäcklund, “Über flachen Transformationen,” Mathematische Annalen, vol. 9, pp. 297-320, 1876.

[8] A. V. Bäcklund, "Zur theorie der Flachentransformationen," Mathematische Annalen, vol. 19, pp. 387$422,1882$.

[9] J. K. Perring and T. H. R. Skyrme, “A model unified field equation,” Nuclear Physics. B, vol. 31, pp. 550-555, 1962.

[10] A. Barone, F. Esposito, C. J. Magee, and A. C. Scott, “Theory and applications of the Sine-Gordon equation," La Rivista del Nuovo Cimento, vol. 1, no. 2, pp. 227-267, 1971.

[11] J. Frenkel and T. Kontorova, "On the theory of plastic deformation and twinning," Journal of PhysicsUSSR, vol. 1, pp. 137-149, 1939.

[12] G. L. Lamb, Jr., "Analytical descriptions of ultrashort optical pulse propagation in a resonant medium," Reviews of Modern Physics, vol. 43, pp. 99-124, 1971.

[13] B. D. Josephson, "Possible new effects in superconductive tunnelling," Physics Letters, vol. 1, no. 7, pp. 251-253, 1962.

[14] J. L. Fergason and G. H. Brown, "Liquid crystals and living systems," Journal of the American Oil Chemists Society, vol. 45, no. 3, pp. 120-127, 1968.

[15] D. Wang, Elimination Methods, Texts and Monographs in Symbolic Computation, Springer, New York, NY, USA, 2001.

[16] S. K. Liu, Z. Fu, S. Liu, and Q. Zhao, "Jacobi elliptic function expansion method and periodic wave solutions of nonlinear wave equations," Physics Letters A, vol. 289, no. 1-2, pp. 69-74, 2001.

[17] Z. Yan, "The new extended Jacobian elliptic function expansion algorithm and its applications in nonlinear mathematical physics equations," Computer Physics Communications, vol. 153, no. 2, pp. 145154, 2003.

[18] W. Malfliet, "Solitary wave solutions of nonlinear wave equations," American Journal of Physics, vol. 60, no. 7, pp. 650-654, 1992.

[19] C. Yan, "A simple transformation for nonlinear waves," Physics Letters A, vol. 224, no. 1-2, pp. 77-84, 1996.

[20] J.-H. He and X.-H. Wu, "Exp-function method for nonlinear wave equations," Chaos, Solitons $\mathcal{E}$ Fractals, vol. 30, no. 3, pp. 700-708, 2006.

[21] E. Fan, "Uniformly constructing a series of explicit exact solutions to nonlinear equations in mathematical physics," Chaos, Solitons and Fractals, vol. 16, no. 5, pp. 819-839, 2003.

[22] Y.-Z. Peng, "Exact solutions for some nonlinear partial differential equations," Physics Letters A, vol. 314, no. 5-6, pp. 401-408, 2003.

[23] Y. Chen and Q. Wang, "Multiple Riccati equations rational expansion method and complexiton solutions of the Whitham-Broer-Kaup equation," Physics Letters A, vol. 347, no. 4-6, pp. 215-227, 2005.

[24] E. Yomba, "Generalized hyperbolic functions to find soliton-like solutions for a system of coupled nonlinear Schrödinger equations," Physics Letters A, vol. 372, no. 10, pp. 1612-1618, 2008.

[25] E. M. E. Zayed, "New traveling wave solutions for higher dimensional nonlinear evolution equations using a generalized $\left(G^{\prime} / G\right)$-expansion method," Journal of Physics A, vol. 42, no. 19, Article ID 195202, 13 pages, 2009.

[26] W.-X. Ma, H. Wu, and J. He, "Partial differential equations possessing Frobenius integrable decompositions," Physics Letters A, vol. 364, no. 1, pp. 29-32, 2007.

[27] W.-X. Ma and J.-H. Lee, "A transformed rational function method and exact solutions to the $3+1$ dimensional Jimbo-Miwa equation," Chaos, Solitons \& Fractals, vol. 42, no. 3, pp. 1356-1363, 2009. 


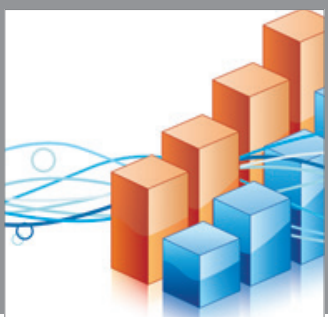

Advances in

Operations Research

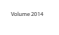

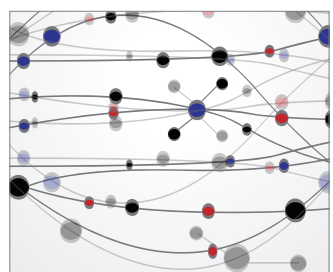

\section{The Scientific} World Journal
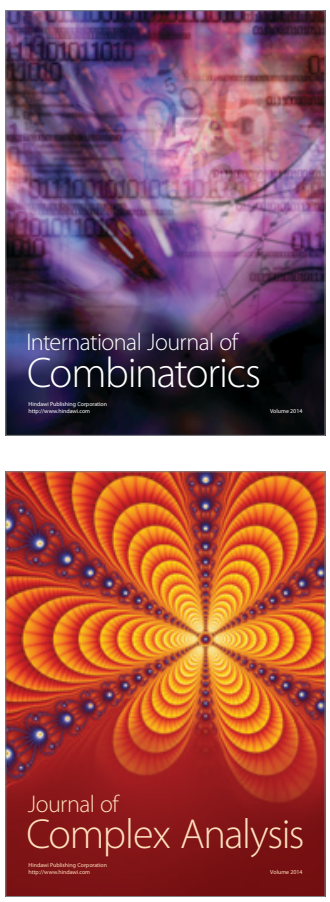

International Journal of

Mathematics and

Mathematical

Sciences
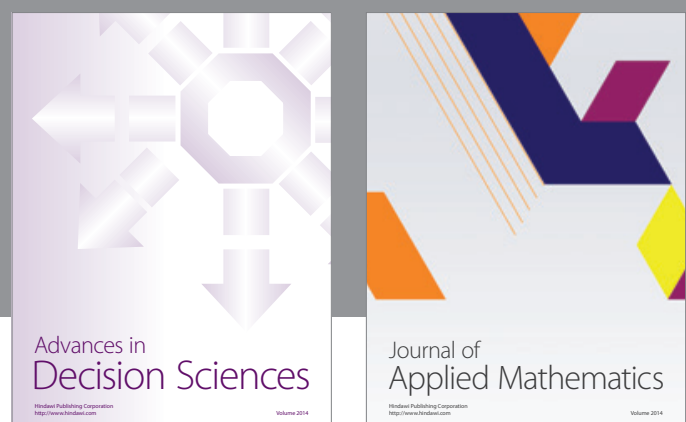

Journal of

Applied Mathematics
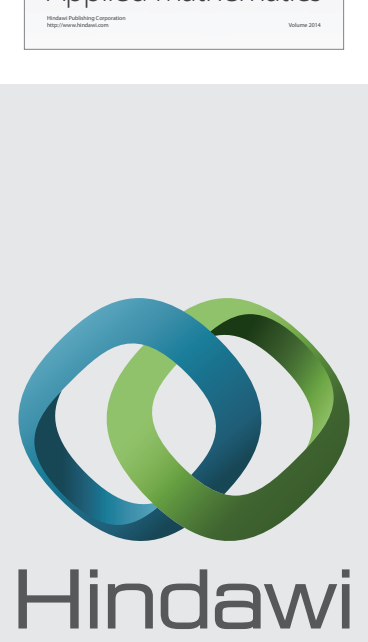

Submit your manuscripts at http://www.hindawi.com
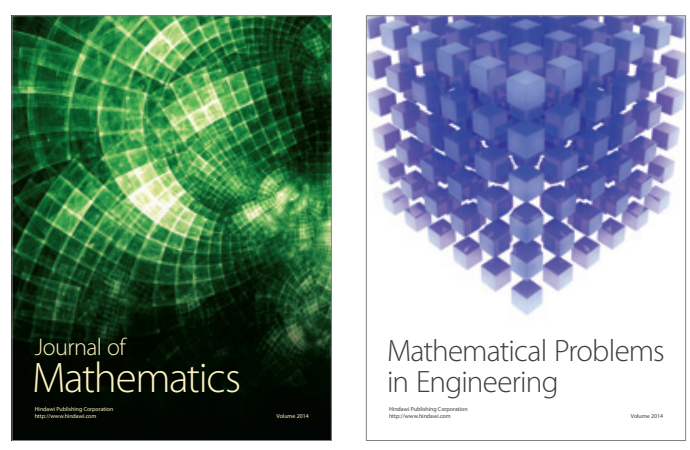

Mathematical Problems in Engineering
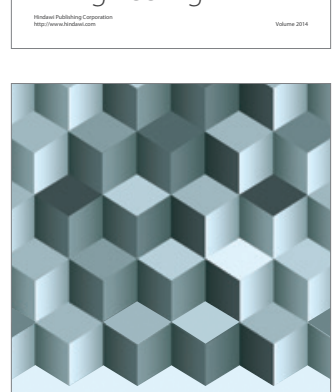

Journal of

Function Spaces
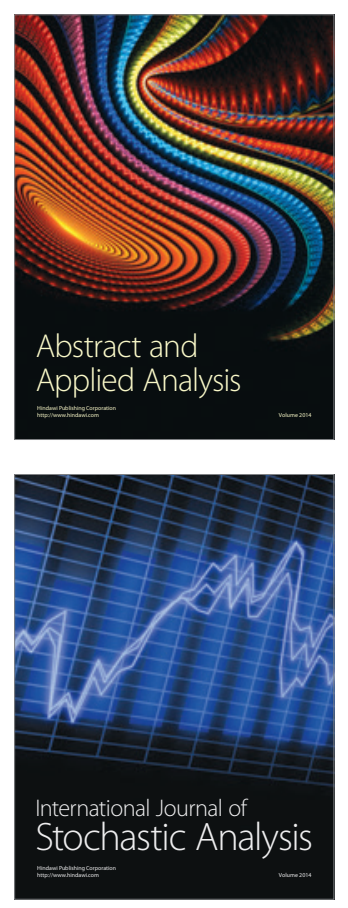

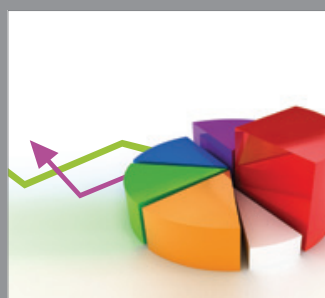

ournal of

Probability and Statistics

Promensencen
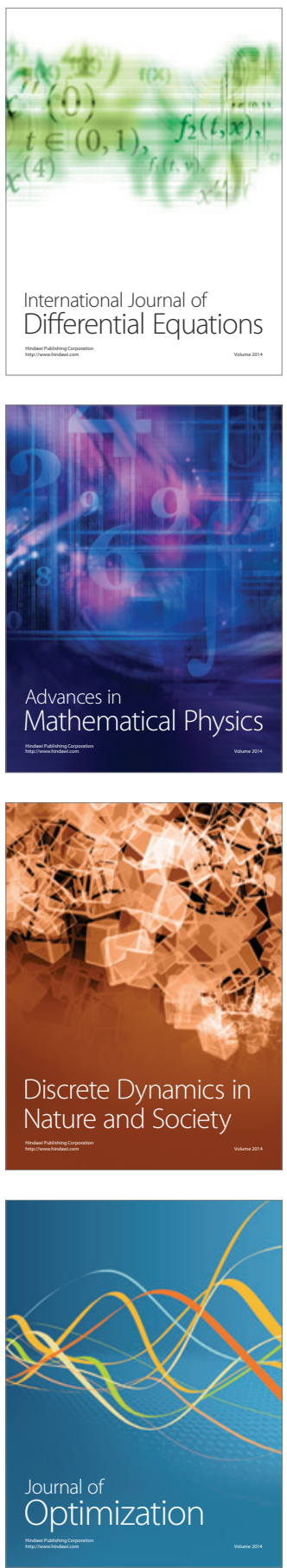